\title{
8. Experiences on Subthalamotomy
}

\author{
Keiji Sano, Masumi Yoshioka, Motohide Ogashiwa, \\ Buichi IshiJImA and Chihiro OHYE \\ Department of Neurosurgery, Faculty of Medicine, University of Tokyo
}

\begin{abstract}
Recently, we have had considerable experiences in the technique of subthalamotomy, especially the stereotactic destruction of the Forel $\mathrm{H}$ field in the treatment of involuntary movements as introduced by Jinnai, Andy and Spiegel.

The lesion of destruction was aimed at the point, $3 \mathrm{~mm}$ posterior and $3 \mathrm{~mm}$ inferior to the midpoint of intercommisural line in the lateral projection and $8-12 \mathrm{~mm}$ lateral to the midline in the anterio-posterior view. The target point was confirmed by stereotactic calculations on the pneumoventriculograms, evoked potential studies of the EEG and EMG changes.

Evoked potentials obtained by stimulation of the region of subthalamus revealed the characteristic recording of slow potentials with long duration and two or three negative peaks followed by one stimulation.

On high cycle stimulation of the subthalamic region, rigidity of muscles and increase in the intensity of tremors were observed on the contralateral side concurrent with the contraction of sternocleidomastoid and trapezius mucles on the ipsilateral side. In diseases of the involuntary movements such as Pakinsonism and spasmodic torticollis, the same effects were exhibited on high cycle stimulation of the subthalamus.

Till now, subthalamotomy was done on eight patients and it was seen that remarkable improvement occurred in patients with Parkinsonism; and $\mathrm{V}-\mathrm{L}$ thalamotomy and subthalamotomy performed on each side was thought to give rise to satisfactory results in the treatment of involuntary movements.

Discussion to 8.
\end{abstract}

\section{Keizo Matsumoto \\ Dept. of Surgery, Osaka University Medical School}

Forel-H-tomy for epileptics has been performed in our service on eleven cases who had severe daily frequent attack of seizures.

It has been already reported by Jinnai and Nishimoto that the surgical result for grand mal type of seizure was satisfactory. Our recent animal experiment showed that the destruction of Forel-H field raise up the threshold to convulsion induced by metrazol injection into the cortex of the cat brain, and suggested that the surgery could be applied to any form of epileptic seizures. The surgery was done for four epileptics who had had petit mal and psychomotor type of seizure. The results were satisfactory. Immediately 
after the surgery, however, aphasia, loss of consciousness, anorexia, hypotonicity of skeletal muscles and myoclonic jerk of limbs were temporarily noted as complications on three cases of children who underwent bilateral surgeries simultaneously. Therefore, bilateral simultaneous Forel-H-tomy should be avoided. A particular case, nine years old female, who was diagnosed as having cerebral palsy associated with epilepsy and behavior problem, underwent bilateral Forel-H-tomy and posterior hypothalamectomy at the same time. After recovery from the surgery, her seizure attacks subsided and aggressiveness of her character improved without any additional neurological deficits. The result indicated potential usefulness of combination of two basal ganglia surgery for such kind of patients.

\section{V.Im. Thalamotomy for Tremor}

Hirotaro NaRABAYASHI

Dept. of Neurology, Juntendo Medical University

Ventrolateral thalamotomy, specially destruction of its posterior part has been experienced effective for tremor, under the nomination of VLthalamotomy.

The author interpreted this in 15 cases, as Ventralis Intermedius Thalamotomy, depending on the results of very small lesions of usually about 4 to 5 diameters. It is completely non-effective for rigidity and is effective selectively on tremor only. Evoked potentials in this nucleus for stimulating the peripheral nerve (ulnar nerve) was obtained with latency of 12 to 13 milliseconds. High cycle stimulation of this nucleus induced acceleration of tremor (in two thirds of the cases examined) and inhibition in one third of the examined cases.

Rhythmic activity on skull leads for stimulating the VL nucleus was not obtained by stimulating the V.Im. nucleus. 